\title{
Hodge experiment photographs quantified with a color function
}

\author{
J.C. Hodge ${ }^{1 *}$ \\ ${ }^{1}$ Retired, 477 Mincey Rd., Franklin, NC, 28734
}

\begin{abstract}
The Hodge Experiment of the Scalar Theory of Everything (STOE) model is the Fraunhofer pattern from a first mask with a single slit impinges on a second mask. The pattern from a second mask slit and the pattern from an edge that differs only in the removal of one of the mask sides are compared. The second mask provides more to the screen pattern than only blocking light as wave models suggest. The second mask contributes to form the screen image as the STOE model suggests.
\end{abstract}

keywords: diffraction, interference, light, STOE, TOE.

\section{INTRODUCTION}

The Scalar Theory of Everything (STOE) model suggested a photon model of light (Hodge 2012). The STOE model uses classical concepts (Hodge 2016b). A computer toy simulation was developed. The Hodge Experiment was developed to explore the STOE predictions about light. The Hodge Experiment uses a diffraction pattern from a first mask slit to form a coherent light intensity pattern on a second mask (see Fig. 1 and 2). This produces a varying intensity of light across the slit without another physical object to "collapses the wave function" ${ }^{1}$. Moving the slit in the second mask allowed differing light intensity profiles. The model predicted a unique diffraction/interference pattern of light on a screen (Hodge 2015b,c). The effect of edges was also explored (Hodge 2016a, 2017). The Hodge Experiment rejects wave models of light.

The STOE model posits photons cause waves in the plenum that reflect off other matter such as atoms in surfaces. These reflected plenum waves then direct the photon. Therefore, added surfaces such as detectors in an experiment changes the photon's path rather than the Quantum Mechanics model wherein the added surface destroys path knowledge. The model in the simulation is that the mask reflects plenum (ether) waves that are caused by photons between the second mask and screen. The plenum waves then direct the photon's path. The

\footnotetext{
*E-mail: jchodge@frontier.com

${ }^{1}$ Thus avoiding a criticism of the Afshar Experiment.
} 


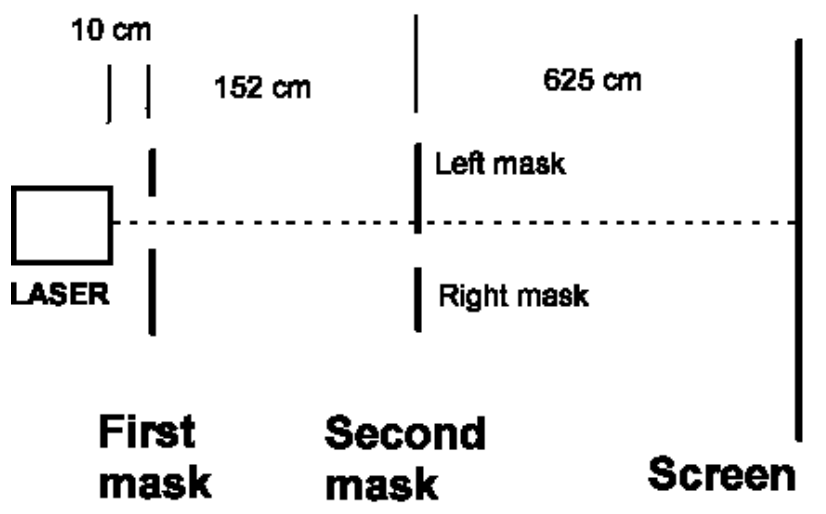

Figure 1: The diagram of the experimental layout.

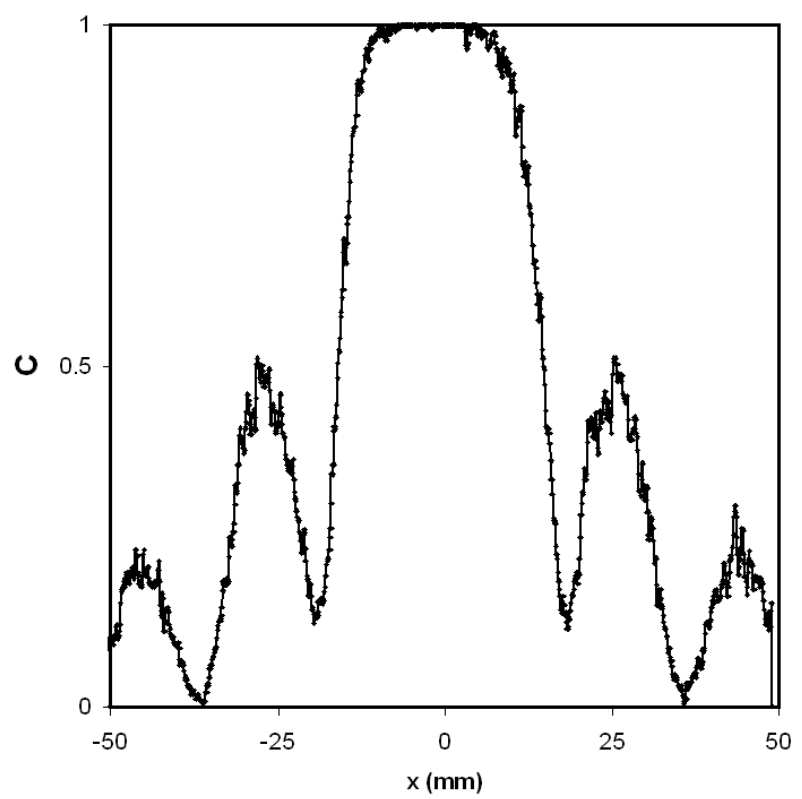

Figure 2: The screen pattern from the first mask, only. 


\section{EXPERIMENT}

gap in the mask allows the plenum waves to pass and not reflect. This incorporates the slit characteristics onto the photon's path and, hence, the screen pattern.

An extension of the Hodge Experiment using a transparent mask suggested a mechanism of interference (Hodge 2017). Another observation of these experiments is that the two-mask arrangement produces interference patterns. That is, the arrangement is a double-slit experiment. Therefore, the observed pattern on a screen is a diffraction pattern as an envelope of interference fringes (see Jenkins and White (1957, p. 314)). The Hodge Experiment is a double-slit, interference experiment. A model of the Hodge Experiment that lacks interference fringes is incorrect. Because all the light passes through both slits, the which-way controversy is irrelevant. Because the second mask is required to produce the interference pattern, the removal of one side of the mask changes the interference pattern. The wave models of diffraction are that the mask only blocks light.

The experiments reported in Hodge (2012) showed the experiment and model produced the same results as the Fraunhofer wave model in the Fraunhofer zone with a uniform illumination across the second mask slit. The derivation of the Fraunhofer model from the STOE model was discussed in Hodge (2015a) which also showed why the mysterious Fresnel-Huygens assumptions were necessary for wave models. The Fraunhofer model that requires uniform illumination across the slit doesn't apply to the Hodge Experiment.

Dr. Kazufumi Sakai (Sakai 2018) suggested quantitative results should be done from photographs although the evaluation may not linearly relate to intensity. But it may allow the "step" measure in the simulation to be correlated to millimeters.

This Paper responds to Dr. Sakai by noting the comparison of wave and STOE models of two experiments with particular attention to the differences caused by the mask. Section 2 describes the experiment. The interpretation is in Section 3. The Discussion and Conclusion are in section 4.

\section{Experiment}

The experimental configuration was the same as in Hodge (2015c). Figure 1 is a diagram of the experimental arrangement. The laser light was $650 \pm 10 \mathrm{~nm}$ and less than $5 \mathrm{mw}$. The first slit was $0.305 \mathrm{~mm}$ wide as determined by a feeler gauge. The photographs in the figures have been converted to gray scale for printing. Uncertainty is $\pm 5 \%$ unless otherwise stated.

The procedure was to photograph the images with a CCD camera, to select a slice of the image, and to evaluate the slice with a Visual Basic program using the "point(x,y)" function. The "point(x,y)" function returns the RGB color number $C$ where $x$ is the horizontal position and $y$ the vertical position of the point evaluated. For each $x$ position, $5 y$ positions were averaged to obtain one $x$ result. The center of the screen image in Fig. 2 is marked on the screen as $x=0 \mathrm{~mm}$ for reference in the other images. 


\section{EXPERIMENT}

There are several characteristics to remember when using this procedure. Figure 3 shows an example. The photographic slice is shown in the top image rendered in grayscale for printing. The second mask slit was $1 \mathrm{~mm}$ wide and placed as depicted in the smaller image at top right of the graph of the first mask's image. The graph is the result of "point $(\mathrm{x}, \mathrm{y})$ " program applied to the slice. The slice includes a central bright spot and several fringes just to the right and 2 other fringes to the left of the bright spot - the interference pattern. Note the small peaks in the graph at the position of the fringes marked with arrows. This demonstrates the "point $(\mathrm{x}, \mathrm{y})$ " function appearance of fringes. However, the graph fails to show the interference fringes close to the center and does detect the fringes farther to the right. The height of the fringe peaks is small relative to the center. The "point $(\mathrm{x}, \mathrm{y})$ " function detects zero when there is no color to detect.

The graph of Fig. 2 shows the point(x,y) measurements for a Fraunhofer pattern. The first secondary intensity maximum should be approximately $5 \%$ of the central intensity maximum. However, if there is even a hint of color from the CCD, the "point( $\mathrm{x}, \mathrm{y})$ " function seems to overreact. Remember it is measuring color, not intensity. That is, it is poor at detecting the intensity but excellent at detecting no light (the minimums of interference patterns). The heights of the graph values are a non-linear indication of intensity.

Starting from the position of the second mask in Fig. 3, the second mask is moved slowly to the right to a point such that the left $(x<0)$ part of the screen image is minimal. The procedure in previous papers was to position the right mask edge to the minima of the first mask. That is, the right part of the slit is at a minima and the intensity of light from the first mask decreases across the second mask slit.

Figure 4 shows the comparison of the Hodge Experiment with a slit width of $2.3 \mathrm{~mm}$ (designate $\mathrm{A}, \mathrm{C}$, and $\mathrm{E}$ in the lower right of each graph) and the edge formed by removal of the right part of the mask (B, D, and F). The top row (A and $\mathrm{B}$ ) shows the result of the point $(\mathrm{x}, \mathrm{y})$ function on the pattern produced on the screen. The middle row shows the calculation of a wave function intensity:

$$
I=\left(f(x) \sum_{i} A_{i} e^{i k r_{i}} / r_{i}\right)^{2}
$$

where $k$ is $2 \pi$ /wavelength and $r_{i}$ is the distance between the $i^{\text {th }}$ Huygens wavelet source point in the slit and the screen $x$ position, $A_{i}$ is the amplitude of the first mask's pattern at the $i^{t h}$ point of the second mask and $f(x)$ is a normalizing function.

The third row (E and F) shows the STOE simulation calculation from Hodge (2015c) calculated in "steps". Figure 1 of Hodge (2015c) shows the photons approaching the mask changing direction toward the edge of the mask. Jenkins and White (1957, p. 379) noted that the diffracting edges appear luminous. The simulation may have had some photons initially directed to near the edge redirected to appear to have slightly higher intensity than zero and hence cause the few photon to the left. Therefore, the simulation was rerun with a 0.01 step 


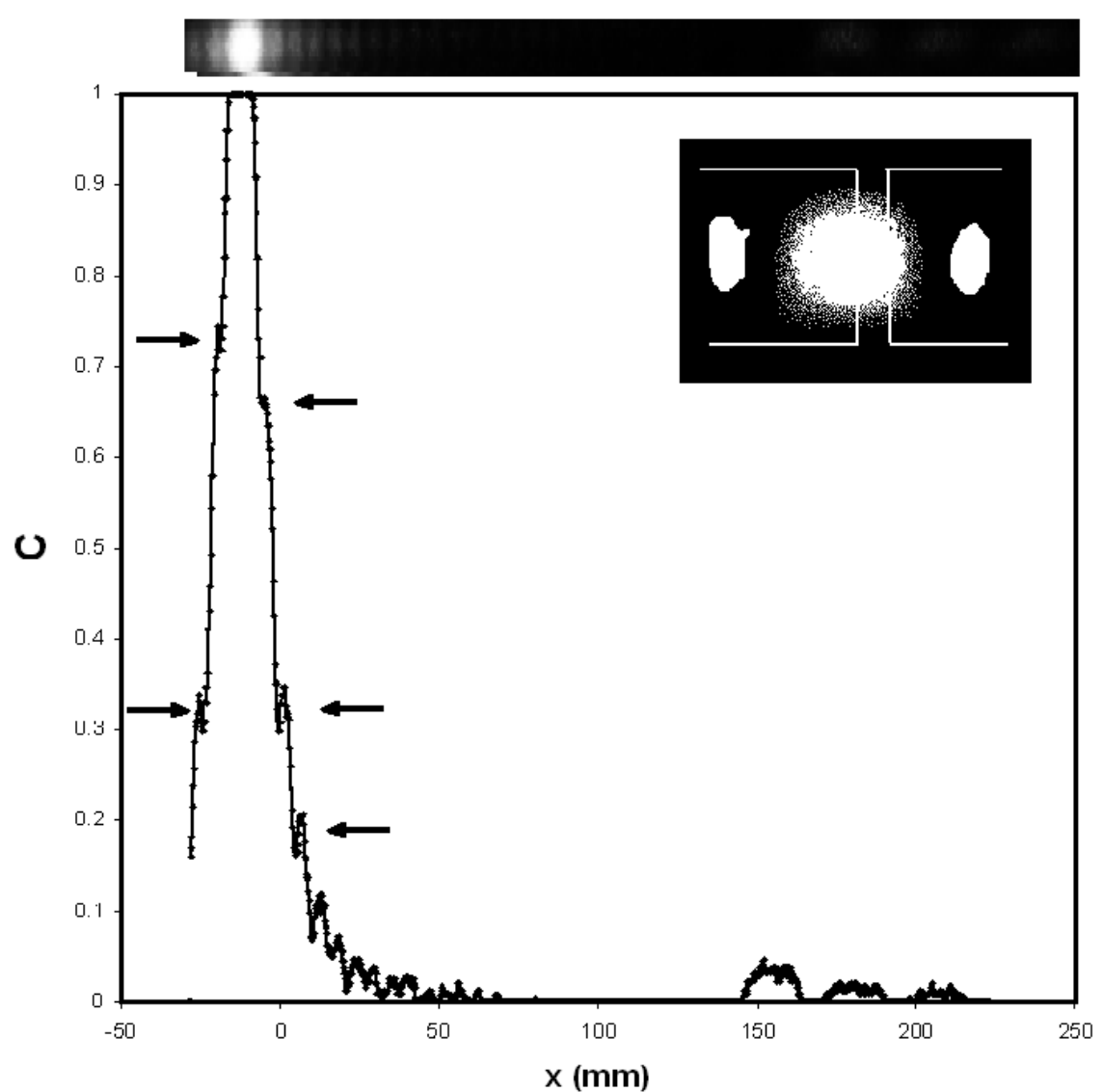

Figure 3: An example showing the mask position relative to the first mask image on the second mask. The top photographic image shows the diffraction pattern and interference fringes relative to the graphical result of the "point(x,y)" function. The arrows mark the fringes. 
change in the position of the initial photons. The result is Fig. 4E.

\section{Interpretation}

The graph of Fig. 4A shows a interference pattern for $x>0 \mathrm{~mm}$. The small spikes appear as interference fringes as in Fig. 3. For $x<0 \mathrm{~mm}$, the decline is relatively rapid compared to Fig. $4 \mathrm{~B}$.

The graph of Fig. 4B to the right of the first minima at approximately $x>19$ $\mathrm{mm}$ is the result of the diffraction pattern from the first mask as seen in Fig. 2. That is, the second mask had no effect on $x>19 \mathrm{~mm}$. For $0 \mathrm{~mm}<x<19$ $\mathrm{mm}$ the $C$ is saturated and shows no detail. The graph to the left $(x<0 \mathrm{~mm})$ is the result of that part of the first mask diffraction pattern that was in the slit of Fig. 4A. This is similar to the diffraction pattern from an edge using the Fresnel method.

Comparing the wave models of Fig. $4 \mathrm{C}$ and D shows only slight difference. However, the interference fringes are more pronounced in Fig. 4C. The major difference between Figs. 4A and B is the presents of the right side mask. Wave models are a poor match for the observed patterns and the differences between Figs. 4A and B. The STOE model uses the mask as a reflecting surface in addition to a blocking surface as seen in Fig. $4 \mathrm{E}$ and F. The STOE model is a better match for the observed screen pattern.

\section{Discussion and Conclusion}

The point $(\mathrm{x}, \mathrm{y})$ function is inadequate to describe the intensity profile of the screen images. The intensity of the light at $x<0$ may be much lower for Fig. 4B as suggested by Fig. 4A. Therefore, a means to measure intensity such as a photon counter may be needed fully show intensity details.

The STOE model needs a relation to calibrate the "step" with the millimeter measures. The "point $(\mathrm{x}, \mathrm{y})$ " function may be adequate.

The Hodge Experiment of the Scalar Theory of Everything (STOE) model is the Fraunhofer pattern from a first mask with a single slit impinges on a second mask. The pattern from a second mask slit and the pattern from an edge that differs only in the removal of one of the mask sides are compared. The second mask provides more to the screen pattern than only blocking light as wave models suggest. The second mask contributes to form the screen image as the STOE model suggests. 

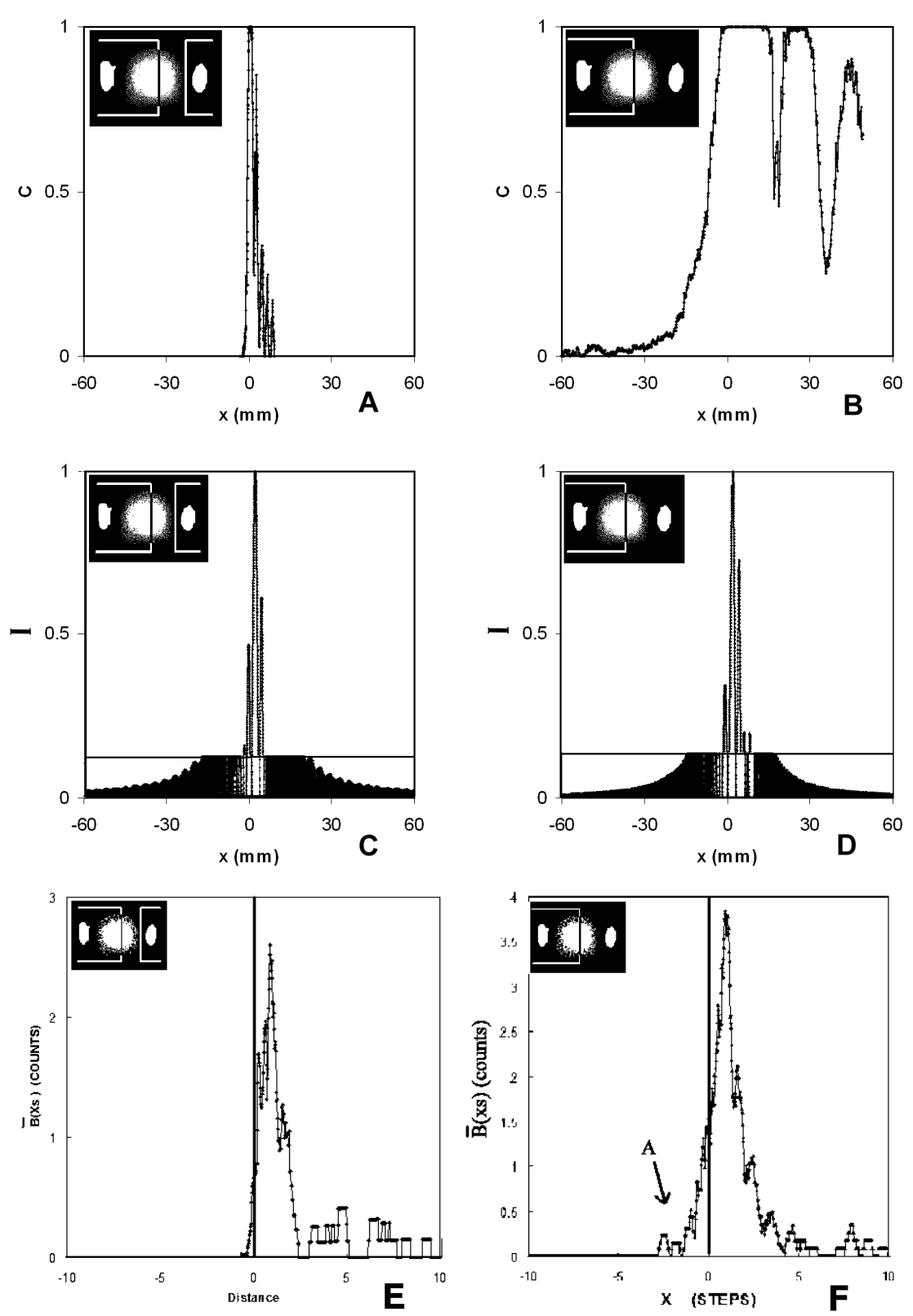

Figure 4: Comparison of actual and model calculations. 


\section{REFERENCES}

\section{References}

Hodge, J.C., 2012, Photon diffraction and interference, IntellectualArchive, Vol.1, No. 3, P. 31, http://intellectualarchive.com/?link=item\&id=597 http://viXra.org/abs/1709.0210

Hodge, J.C., 2015a, Single Photon diffraction and interference , IntellectualArchive, Vol.4, No. 4, P. 1, http://intellectualarchive.com/?link=item\&id=1557

Hodge, J.C., 2015b, Light diffraction experiments (continued) , http://intellectualarchive.com/?link=item\&id=1594

Hodge, J.C., 2015c, Diffraction experiment and its STOE photon simulation program rejects wave models of light , IntellectualArchive, Vol.4, No. 6, P.11 , http://intellectualarchive.com/?link=item\&id=1603 http://viXra.org/abs/1510.0347

Hodge, J.C., 2016a, Hodge Experiment distinguishes between wave and particle caused diffraction patterns, IntellectualArchive, Vol.5, No. 3, P. $7, \quad$ http://intellectualarchive.com/?link=item\&id=1712 http://viXra.org/abs/1604.0358

Hodge, J.C., 2016b, STOE assumptions that model particle diffraction and that replaces $Q M$, IntellectualArchive, Vol.5, No. 3, P. 1, http://intellectualarchive.com/?link=item\&id=1719

Hodge, J.C., 2017, Hodge Experiment (continued) of interference with a slit in a transparent mask rejects wave models of light , http://intellectualarchive.com/?link=item\&id=1862 http://viXra.org/abs/1708.0383

Jenkins, F. A. and White, H. E., Fundamentals of Optics, (McGraw-Hill Book Company, Inc., New York, NY, USA, 1957)

Sakai, K., 2018, personal communication. 